Original Article

\title{
FEASIBILITY OF AN INTENSIVE CONTROL INSULIN-NUTRITION GLUCOSE MODEL 'ICING' WITH MALAYSIAN CRITICALLY-ILL PATIENT
}

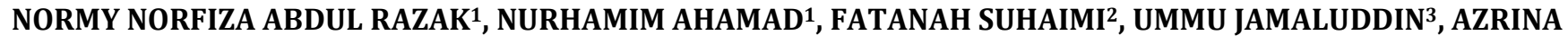 \\ M. RALIB ${ }^{4}$
}

${ }^{1}$ Universiti Tenaga Nasional, Kajang, Selangor, ${ }^{2}$ Universiti Sains Malaysia, Kepala Batas, Pulau Pinang, ${ }^{3}$ Universiti Malaysia Pahang, Pekan, Pahang, ${ }^{4}$ Universiti Sains Islam Antarabangsa, Kuantan, Pahang

Email: normy@uniten.edu.my

Received: 24 Apr 2016 Revised and Accepted: 29 May 2016

\begin{abstract}
A clinically verified patient-specific glucose-insulin metabolic model known as ICING is used to account for time-varying insulin sensitivity. ICING was developed and validated from critically-ill patients with various medical conditions in the intensive care unit in Christchurch Hospital, New Zealand. Hence, it is interesting and vital to analyse the compatibility of the model once fitted to Malaysian critically-ill data. Results were assessed in terms of percentage of model-fit error, both by cohort and per-patient analysis. The ICING model accomplished median fitting error of $<1 \%$ over data from 63 patients. Most importantly, the median per-patients is at a low fitting error of $0.34 \%$ and per cohort is $0.35 \%$. These results provide a promising avenue for near future simulations of developing tight glycaemic control protocol in the Malaysian intensive care unit.
\end{abstract}

Keywords: Glucose-insulin model, TGC, Malaysian critically-ill, model-based control.

(C) 2016 The Authors. Published by Innovare Academic Sciences Pvt Ltd. This is an open access article under the CC BY license (http://creativecommons.org/licenses/by/4.0/) DOI: http://dx.doi.org/10.22159/ijpps.2016v8s2.15218.

\section{INTRODUCTION}

Critical care units are seeing rapidly growing numbers of patients with hyperglycaemia (high blood glucose) which is similar to diabetes due to endemic insulin resistance as well as the stress of their condition. The metabolic response to stress is characterised by major changes in glucose metabolism. Increased secretion of counter-regulatory hormones leads to a prominent rise in endogenously produced glucose and the rate of hepatic gluconeogenesis, as well as a reduction in insulin sensitivity. An essential treatment of hyperglycaemia in all cases is to maintain active insulin control by monitoring glucose levels and supplying insulin as necessary. In the critical care setting it is even more critical as active control has been shown to reduce mortality by up to $45 \%$ [1]. Inhibiting the physiological response to increased glycaemic levels are factors such as increased insulin resistance, absolute or relative insulin deficiency, and drug therapy. Hence, it is vital that normoglycaemia within the intensive care unit (ICU) is maintained.

Hyperglycaemia is not only a marker for severity of illness, it also worsen outcomes, leading to an increased risk of further complications, such as severe infections, myocardial infarctions, polyneuropathy and multiple-organ failure [2]. Done effectively, active insulin control in the ICU reduces organ failure, save lives and reduces cost. However, effective insulin control has proven difficult, particularly within increasingly tight nursing resources as many unit employ less than one nurse per patient. A model-based approach offers the opportunity to improve productivity and enhance the consistency and quality of insulin control to yield successful results [1] Of the prior successful, and many unsuccessful insulin studies studies, none employed computational model-based control to manage the patient's glycaemic level during or after disaster occurred. Thus, there is significant motivation for effective maintenance of normal glucose levels which have shown potential to provide clinically effective solutions [3-5].

In the ICU, catastrophic disasters may force difficult decisions for intensivist and nurses when demand for round-the-clock care greatly exceeds available resources. Most critical care units routinely function at or near capacity, significantly decreasing available critical care response capabilities for disasters. Current hospitals recognize that excess critical care capacity for a disaster is extremely limited. Critically ill patients often experience physiological stress responses which lead to a highly complex and dynamic metabolic state, making effective treatment of hyper-and hypo-glycaemia difficult. Glycaemic variability, and thus poor control, is also independently associated with an increase in mortality. The critical care response to disaster will be challenged and patients care might shift from individually focused to whole group.

This study targets to design a model-based approach towards safe management of hyperglycaemia for Malaysian critically-ill patients. However, the focus of this paper is to assess the compatibility of a clinically verified glucose-insulin model with Malaysian critically-ill data. In the first part of this paper, an analysis of the efficacy of current protocol practice is presented. Development of time-varying profiles follows suit as these are used as the critical marker of a patient's metabolic state. The insulin sensitivity metric is independent of both the insulin and nutrition inputs used to derive it. Identifying insulin sensitivity requires capturing the fundamental dynamics of the glucose regulatory system. A clinically verified patient-specific glucose-insulin metabolic model known as ICING [6] is used to account for time-varying insulin sensitivity. ICING [6] was developed and validated from critically-ill patients with various medical conditions in the intensive care unit in Christchurch Hospital, New Zealand. Hence, it is interesting and vital to analyze the compatibility of the model once fitted to Malaysian critically-ill data. Results were assessed in terms of percentage of model-fit error, both by cohort and per-patient analysis. The ICING model [6] accomplished median fitting error of $<1 \%$ over data from 63 patients. Most importantly, the median perpatients is at a low fitting error of $0.34 \%$ and by cohort is $0.35 \%$. These results provide a promising avenue for near future simulations of developing TGC protocol in the Malaysian intensive care unit.

\section{MATERIALS AND METHODS}

\section{Methodology}

Data of 63 critically-ill patients were gathered from the intensive care unit of Hospital Tengku Ampuan Afzan Kuantan (HTAA), Pahang. The socio-demographic characteristics and cohort details are summarized in table 1 . Malay ethnicity makes the largest cohort at $87 \%$ and percentage of male patients is $59 \% .67 \%$ of patients are under medical category and $67 \%$ of patients fall under age cohort of over $50 \mathrm{y}$ old. The Intensive Insulin Protocol used in HTAA to maintain the blood glucose (BG) concentration target was set at 5.1-8.0 mmol/l. Ethics was granted by IIUM Research Ethics Committee and National Institute of Health (NIH) 
Table 1: Socio-demographic characteristics

\begin{tabular}{ll}
\hline Socio-demographic characteristics & Total $\mathbf{N = 6 3}$ \\
\hline Gender & \\
Female & 26 \\
Male & 37 \\
Mortality & 24 \\
Dead & 38 \\
Alive & \\
Referring Unit Category & 1 \\
Neuro & 20 \\
Surgical & 42 \\
Medical & \\
Age groups (years) & 4 \\
$<29$ & 6 \\
$30-39$ & 11 \\
$40-49$ & 42 \\
$>50$ & \\
Ethnicity & 1 \\
Indian & 3 \\
Others & 4 \\
Chinese & 55 \\
Malays &
\end{tabular}

\section{System model}

The glucose-insulin physiological model used in this study is clinically-validated [6]. Known as ICING model, it uses previous and current BG values, previous nutrition and previous insulin doses to compute the insulin sensitivity, SI of the patient over the previous time period, based on parameter identification algorithm [7] which fits the model to the clinically observed behavior. The resulting time-varying SI profiles represent time-varying metabolic status for individual patients. Model accuracy is assessed by percentage of fitting error, where fitting error is the error between the measured and the modelled blood glucose levels. Testing new interventions with this profile in simulations provides new outputs. Thus, the profile of SI can be used to create "virtual patients" for testing insulin protocols. Virtual simulations for protocol testing will be carried out in future for controller model development. Analysis is done on by cohort which reads the statistics on the possible hourly fitting errors (weighing each hour equally) while per-patient is statistics on each individual patient (weighing each hour equally).

Equations (1) to (6) are defined in the nomenclature of table 2.

Table 2: ICING equations description

\begin{tabular}{llll}
\hline Symbols & Descriptions & Symbols & Descriptions \\
\hline $\mathrm{G}(\mathrm{mmol} / \mathrm{l})$ & Total plasma glucose & $\mathrm{V}_{\mathrm{G}}(\mathrm{L})$ & Glucose distribution volume \\
$\mathrm{G}_{\mathrm{E}}(\mathrm{mmol} / \mathrm{l})$ & Blood glucose at equilibrium level & $\mathrm{P}(\mathrm{t})(\mathrm{mmol} / \mathrm{min})$ & External nutrition \\
$\mathrm{I}(\mathrm{mU} / \mathrm{l})$ & Plasma insulin & $\alpha_{\mathrm{I}}(\mathrm{L} / \mathrm{mU})$ & Saturation plasma insulin disappearance \\
$\mathrm{Uex}(\mathrm{t})(\mathrm{mU} / \mathrm{min})$ & Exogenous insulin input & $\alpha_{\mathrm{G}}(\mathrm{L} / \mathrm{mU})$ & Saturation of insulin-simulated glucose removal \\
$\mathrm{Q}(\mathrm{mU} / \mathrm{l})$ & Interstitial insulin & $\mathrm{d}_{1} \mathrm{~d}_{2}\left(\mathrm{~min}^{-1}\right)$ & Transport rate \\
$\mathrm{k}\left(\mathrm{min}^{-1}\right)$ & Effective life of insulin in system & $\mathrm{P} 2(\mathrm{mmol} / \mathrm{min})$ & Represent gut \\
$\mathrm{P}_{\mathrm{G}}\left(\mathrm{min}^{-1}\right)$ & Patient endogenous glucose removal & $\mathrm{D}(\mathrm{t})(\mathrm{mmol} / \mathrm{min})$ & Dextrose amount from enteral feeding \\
$\mathrm{SI}\left(\mathrm{L} / \mathrm{mU}^{\mathrm{min}}\right)$ & Interstitial insulin & $\mathrm{CNS}(\mathrm{mmol} / \mathrm{min})$ & Uptake central nervous system \\
$\mathrm{VI}(\mathrm{L})$ & Insulin distribution value & $\mathrm{n}_{\mathrm{I}}(\mathrm{min}-1)$ & Diffusion constant insulin between compartment \\
$\mathrm{P} 1(\mathrm{mmol} / \mathrm{min})$ & Represent stomach & $\mathrm{n}_{\mathrm{K}}(\mathrm{min}-1)$ & Kidney clearance rate of insulin from plasma \\
$\mathrm{PN}(\mathrm{t})$ & Parenteral dextrose & $\mathrm{n}_{\mathrm{L}}(\mathrm{min}-1)$ & Liver clearance rate of insulin from plasma \\
$\mathrm{EGP}(\mathrm{mmol} / \mathrm{min})$ & Production endogenous glucose & $\mathrm{n}_{\mathrm{C}}(\mathrm{min}-1)$ & Cellular insulin clearance rate from interstitium \\
\hline
\end{tabular}

\section{Total plasma glucose}

Interstitial insulin

$$
G(t)=-p G G(t)-S I(G(t)) \frac{Q(t)}{1+\alpha G Q(t)}+\frac{P(t)+E G P b-C N S}{V G}
$$

$$
\begin{aligned}
& Q(t)=n I(I(t)-Q(t))-n C \frac{Q(t)}{1+\alpha G Q(t)} \\
& I(t)=-n K I(t)-n L \frac{I(t)}{1+\alpha I I(t)}-n I(Q(t)+I(t))+\frac{\operatorname{uex}(t)}{V I}+(1-x L) \frac{\text { uen }(G)}{V I} \text {. }
\end{aligned}
$$

$$
\begin{aligned}
& P 1=-d 1 P 1+D(t) . \\
& P 2=-\min (d 2 P 2, P \max )+d 1 P 1
\end{aligned}
$$$$
P(t)=\min (d 2 P 2, P \max )+P N(t)
$$

\section{RESULTS AND DISCUSSION}

Fig. 1 shows the cumulative distribution frequency (CDF) of BG measurement levels for 63 patients undergoing intensive insulin therapy in HTAA, as per cohort (fig. 1a) and per-patient (fig. 1b). The median of BG measurement levels by cohort is at $8.0 \mathrm{mmol} / \mathrm{l}$ with an interquartile range of $[6.4,10.1] \mathrm{mmol} / \mathrm{l}$. Fig. 2 shows a random sample of patient profile. The first panel depicts the $\mathrm{BG}$ measurement level while the $2^{\text {nd }}$ panel shows the insulin infusion received by the patient. The $3^{\text {rd }}$ and last panel represents the nutrition received and the hourly SI profiles of the patient. SI profiles here are the only metabolic indicator of the patient, an output from ICING model. From this study, 63 SI profiles have been generated. These profiles represent virtual patients which is important for the model-based controller development, an on-going research but not the focus of this paper. In fig. 3, CDF of model fit error for the overall patients fitted to the ICING [6] model is shown. The model achieved a low error at $0.34 \%$ perpatient and $0.35 \%$ by cohort.

From fig. 1, it can be concluded that $50 \%$ of critically-ill patients in the ICU still have unsatisfactory control quality in their BG measurement levels. Half of the patients recorded BG levels above $8 \mathrm{mmol} / \mathrm{l}$ even the target was set at $5.1-8.0 \mathrm{mmol} / \mathrm{l}$. This drives the motivation to implement a model-based control in a Malaysian ICU setting. Modelbased control provides a more systematic approach in the management of hyper glycaemia, more so in a high-occupancy rate ICU. Rather than using the concept of 'treat' to meet target, modelbased approach 'prevents' to safely manage BG within desired level.

Patients in the critical settings have more variable, dynamic and unpredictable BG. By using model-based, patient's hourly metabolic indicator can be calculated in real-time. Hence, any interventions, for example the amount of insulin or nutrition to be given would be based upon this $S I$ indicator. Therefore, all treatments will be tailored to each patient unlike a one-size fits all method. Sliding-scale insulin protocol as normally seen in a lot of hospital settings uses a standardized care across patients. More often than not, insulin is the only means of control. ICING [6] on the other hand, uses both insulin and nutrition to predict the required BG for the next hour and up to $4 \mathrm{hr}$. Based upon the low percentage of model-fit error, it is promising that ICING [6] model is suitable and relevant to be used for Malaysian critically-ill patients. However, this is a proof of concept and a pilot trial is still needed to validate clinically. This study can be expanded to many other areas. For example, control in high occupancy rate where intervention frequency would be further apart.

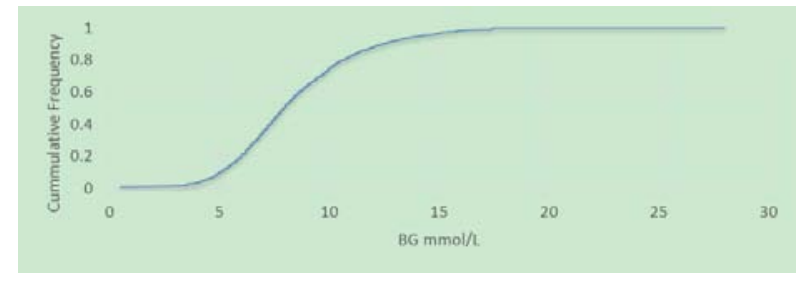

Fig. 1a: Per-cohort 


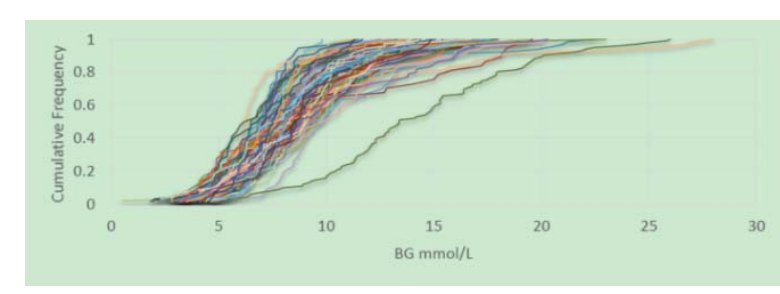

Fig. 1b: Per-patient

Fig. 1: CDF for BG measurement levels (mmol/l) of by cohort and per-patient

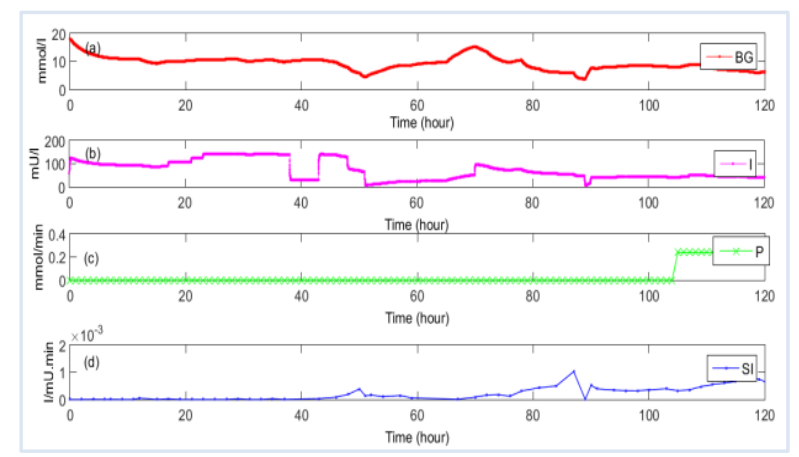

Fig. 2: A random patient profiles receiving intensive insulin therapy in a Malaysian ICU setting. Top panel represents the BG measurement level ( $\mathrm{mmol} / \mathrm{l})$ of the patient, $2^{\text {nd }}$ panel shows the insulin infusion received $(\mathrm{mU} / \mathrm{l})$ during ICU stay, $3^{\text {rd }}$ panel is the nutrition received ( $\mathrm{mmol} / \mathrm{min})$ and the last panel is the $S I$ profiles generated from the ICING model
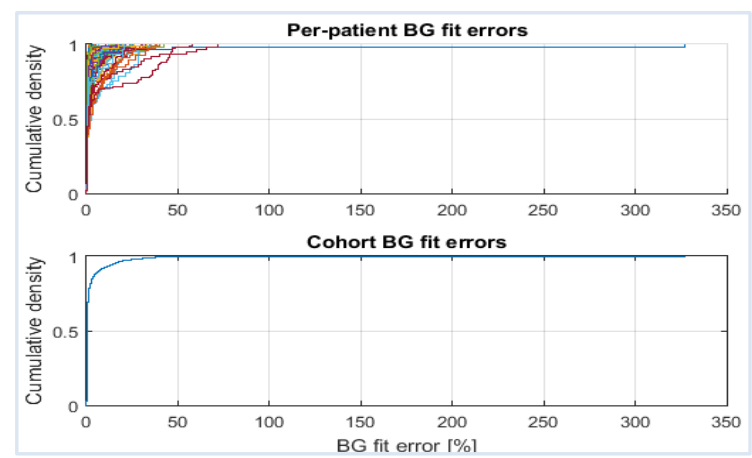

Fig. 3: Top panel shows the per-patient CDF of percentage model fit error (\%) of bottom panel gives the by cohort model fit error

\section{CONCLUSION}

The intensive glucose-nutrition insulin model, ICING validated in this study with clinical data from a Malaysian ICU setting shows a promising result for model-based control development.

\section{ACKNOWLEDGEMENT}

We are grateful to MOHE, Malaysia for the Fundamental Research Grant Scheme, FRGS that enables a strong collaboration between academics and medical expert.

\section{CONFLICT OF INTERESTS}

Declared none

\section{REFERENCES}

1. JG Chase, AJ Le Compte, F Suhaimi, GM Shaw, A Lynn, J Lin, et al. "Tight glycemic control in critical care-the leading role of insulin sensitivity and patient variability: a review and model-based analysis." Computer Methods Programs Biomed 2011;102:156-71.

2. JS Krinsley. "Glycemic variability: a strong independent predictor of mortality in critically ill patients." Crit Care Med 2008;36:3008-13.

3. JG Chase, AJ Le Compte, JC Preiser, GM Shaw, S Penning, T Desaive. Physiological modeling, tight glycemic control, and the ICU clinician: what are models and how can they affect practice? Ann Intensive Care 2011;1:11.

4. L Ward, J Steel, A Le Compte, A Evans, CS Tan, S Penning, et al. Interface design and human factors considerations for modelbased tight glycemic control in critical care. J Diabetes Sci Technol 2012;6:125-34.

5. AJ Le Compte, DS Lee, JG Chase, J Lin, A Lynn, GM Shaw. Blood glucose prediction using stochastic modeling in neonatal intensive care. IEEE Trans Biomed Eng 2010;57:509-18.

6. J Lin, NN Razak, CG Pretty, A Le Compte, P Docherty, JD Parente, et al. A physiological intensive control insulinnutrition-glucose (ICING) model validated in critically ill patients. Comput Methods Programs Biomed 2011;102:192205.

7. CE Hann, JG Chase, J Lin, T Lotz, CV Doran, GM Shaw. Integralbased parameter identification for long-term dynamic verification of a glucose-insulin system model. Comput Methods Programs Biomed 2005;77:259-70.

How to cite this article

- Normy Norfiza Abdul Razak, Nurhamim Ahamad, Fatanah Suhaimi, Ummu Jamaluddin, Azrina M Ralib. Feasibility of an intensive control insulin-nutrition glucose model 'ICING' with malaysian critically-ILL patient. Int J Pharm Pharm Sci 2016;8 Suppl 2:40-42. 